\title{
On Improving Sine Sweep Impulse Response Measurments through Adaptive Filtering
}

\author{
Valentin Adrian Niță* \\ Telecommunications Department, Politehnica University of Bucharest, Bucharest, Romania
}

\author{
DOI: $\underline{10.36348 / \text { sjet.2021.v06i01.002 }}$ \\ | Received: 13.12.2020 | Accepted: 30.12.2020 | Published: 05.01.2021 \\ *Corresponding author: Valentin Adrian Niţă
}

\section{Abstract}

Sine sweeps proves to be a reliable tool for measuring impulse responses even in noisy conditions or by using loudspeakers which are not linear. In this paper we present some results regarding an enhanced sine sweep method, used for impulse response measurement, based on the use of adaptive filtering. Using MATLAB, the conditions of a real case scenario impulse response measurement are simulated in order to quantify the performances of the proposed method by using as reference the performances of the classical sine sweep method.

Keywords: Adaptive filtering; sine sweep; affine projection algorithm.

Copyright $\odot 2021$ The Author(s): This is an open-access article distributed under the terms of the Creative Commons Attribution 4.0 International License (CC BY-NC 4.0) which permits unrestricted use, distribution, and reproduction in any medium for non-commercial use provided the original author and source are credited.

\section{INTRODUCTION}

Modern computational capabilities make sine sweep a reliable tool for measuring impulse response of a room. We propose an enhanced version of the classical sine sweep method. Different studies regarding classical sine sweep performances can be seen in [1-5].

The paper is organized as follows:

- In Section II a short description of the classical sine sweep method is presented, information regarding particularities of the signals that are used in order to measure impulse responses and specific parameters that should be tuned in order to obtain a steady tool;

- A brief introduction in affine projection algorithms for adaptive filtering can be seen in Section III;
- Section IV present the proposed enhancements for improving classical sine sweep method for measuring impulse response of a room;

- Numerical results can be seen in Section V based on two case scenarios, a simple scenario where we assume a classical matched filtering application with a simple impulse response of the channel with only 4 coefficients different from zero and a second more complex scenario where we test the performances of the enhanced sine sweep method used for room impulse response measurements.

- In the end Section VI concludes the paper.

For comparing the performances of the proposed enhancement in contrast with the performances of the classical sine sweep algorithm we use MSE (mean square error) between the real impulse response and the measured impulse response.

\section{ROOM IMPULSE RESPONSE DETECTION BASED ON SINE SWEEP}

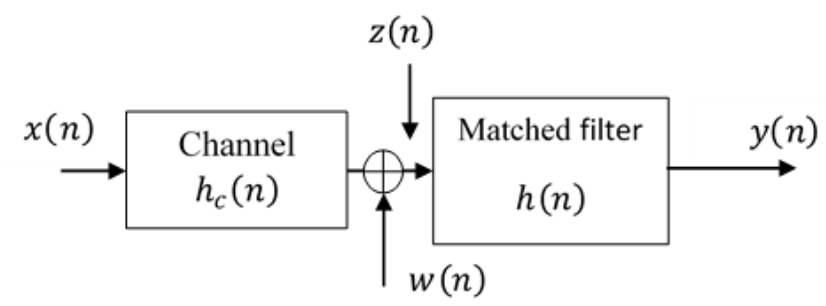

Fig-1: Matched filter configuration 
In Fig-1 the block diagram of a classical matched filter configuration can be seen, which is usually used in radar applications where $x(n)$ is a known chirp signal and

$$
\mathrm{h}(n)=x^{*}(N-n)
$$

Where $\mathrm{N}$ is the number of samples of $x(n)$.

In an impulse response measurement trough sine sweep the block diagram from Fig-1 it changes as follows, the input signal that is passed through the channel to measure the impulse response of the channel based on the output of the matched filter is generated using the following equation [1]:

$$
x(n)=\sin \left[\frac{f_{1} \mathrm{~T}}{\ln \left(\frac{f_{2}}{f_{1}}\right)}\left(e^{2 \pi \frac{n}{\mathrm{~T} F s} \ln \left(\frac{f_{2}}{f_{1}}\right)}-1\right)\right] p_{\mathrm{T} F s}(n)
$$

Where $\mathrm{T}$ - represents the duration of the generated signal, $f_{1}$ is the smallest frequency with which the sweep starts and $f_{2}$ represents the highest frequency obtained at the end of the sweep and $F_{s}$ is the sampling frequency. The band of the measured impulse response will be between $f_{1}$ and $f_{2}$.

In Fig 2 and 3 we can see an example of $x(n)$ waveform, respectively the spectrum for $\mathrm{f} 1=10 \mathrm{~Hz}, \mathrm{f} 2=$ $2000 \mathrm{~Hz}, \mathrm{~T}=4 \mathrm{~s}$ and $\mathrm{Fs}=4000 \mathrm{~Hz}$. We can see that the frequency of the signals is increasing as a function of time, but at the same time the power is decreasing.

The modified matched filter with the input signal must be constructed so that its transfer function should be $H(\omega)=\frac{1}{\mathrm{X}(\omega)}$

In the end the output $\mathrm{y}(n)$ should contain the impulse response of the channel (in our case the room) $h_{c}(n)$.

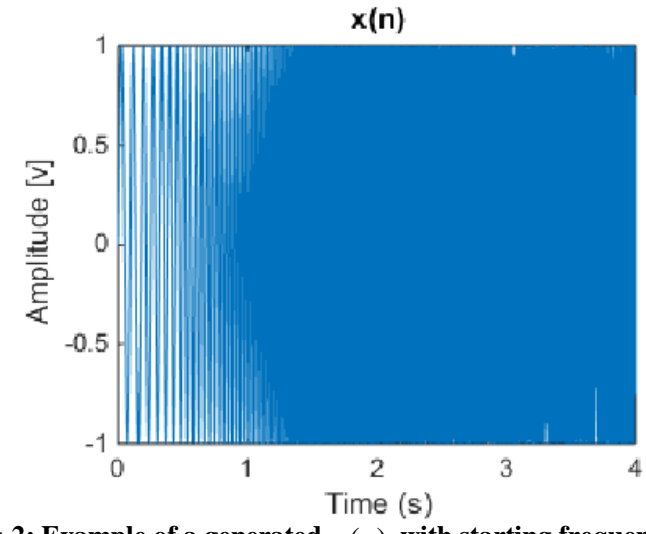

Fig-2: Example of a generated $x(n)$ with starting frequency $10 \mathrm{~Hz}$, end frequency $2000 \mathrm{~Hz}, \mathrm{~T}=4 \mathrm{~s}$ and sampling frequency $4000 \mathrm{~Hz}$

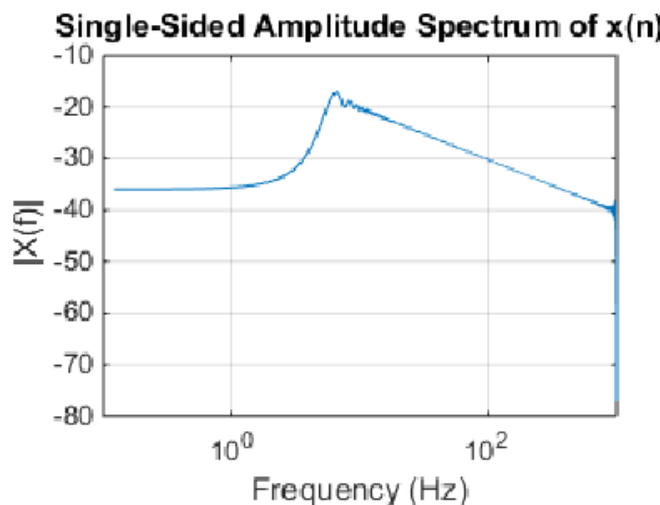

Fig-3: Spectrum of $x(n)$ from Fig-2

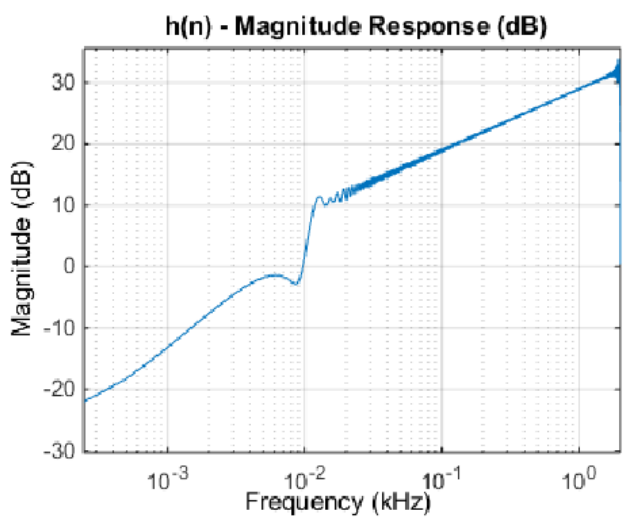

Fig-4: Magnitude response of the filter $h(n)$ matched to the signal from Fig-1

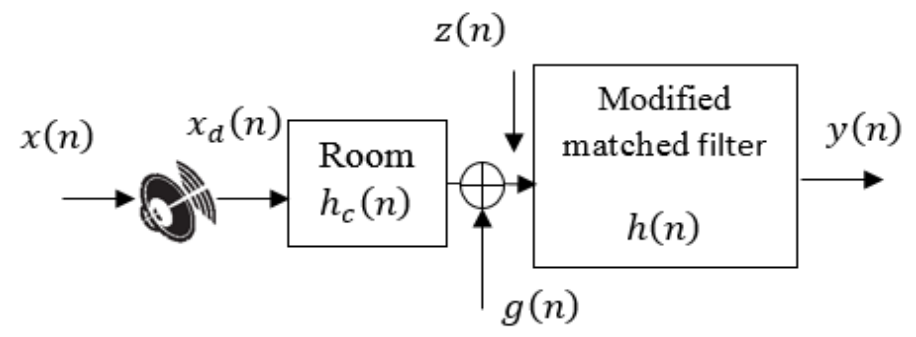

Fig-5: Impulse response measurements configuration including loudspeaker nonlinearities 
In Fig-4 the magnitude response of the matched filter $h(n)$ with the signal generated in Fig-2, used for measuring impulse response of a room.

The classical matched configuration presented in Fig-1, in an impulse response measurement, trough sine sweep method becomes what it can be seen in Fig-5. The signal that enters into the room is not $x(n)$ is a distorted version of it, $x_{d}(n)$, due to the loudspeaker used for playing the signal into the room which is nonlinear. The signal that enters the matched filter is recorded using a microphone. In Fig-5 we can see the system configuration used in the simulation that models also the behavior of the loudspeaker using the Hammerstein model [6].

Based on the Hammerstein model the signal that plays in a room to measure impulse response using sine wave will be

$$
x_{d}(n)=\sum_{k=1}^{N}\left(x^{k} * h_{k}\right)(n)
$$

Where $h_{k}(n)$-represents the Hammerstein model kernels, and $*$ is convolution.

\section{ADAPTIVE AFFINE PROJECTION ALGORITHM IN THE CONTEXT OF SYSTEM IDENTIFICATION}

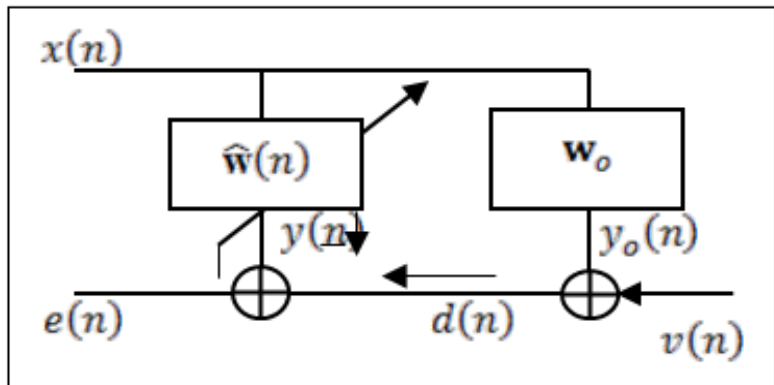

Fig-6: System identification configuration

A system identification configuration is illustrated in Fig-6. The adaptive filter coefficients are changing iteratively until its output is as close as possible with the desired signal output if the systems converge to a steady state.

For an affine projection algorithm (APA) the desired output is given by

$$
d(n)=\mathbf{w}_{\mathbf{0}}^{\mathbf{T}} \mathbf{x}(n)+v(n)
$$

$$
\hat{\mathbf{w}}(n)=\hat{\mathbf{w}}(n-1)+\mu \mathbf{A}^{\mathrm{T}}(n)\left[\mathbf{A}(n) \mathbf{A}^{\mathrm{T}}(n)+\delta \mathbf{I}\right]^{-1} \mathbf{e}(n)
$$

Where

$$
\begin{gathered}
\mathbf{A}^{\mathrm{T}}(n)=[\mathbf{x}(n), \mathbf{x}(n-1), \ldots, \mathbf{x}(n-M+1)], \ldots \ldots \ldots \ldots \ldots . . \\
\mathbf{x}(n-k)=[x(n-k), x(n-k-1), \cdots, x(n-k-N+1)]^{\mathrm{T}}, \\
k=0, \cdots, M-1
\end{gathered}
$$

$\mathbf{d}(n)=\mathbf{A}(n) \mathbf{w}_{0}+\mathbf{v}(n)$

$\mathbf{e}(n)=\mathbf{d}(n)-\mathbf{y}(n)$

$=\mathbf{A}(n) \mathbf{w}_{0}+\mathbf{v}(n)-\mathbf{A}(n) \hat{\mathbf{w}}(n-1)$

$\mathbf{v}(n)=[v(n), v(n-1), \cdots, v(n-M+1)]^{\mathrm{T}}$ 
With: $\mu$ - step size, $\delta$ - regularization parameter, $M-$ projection order, $\mathrm{N}-$ filter order.

Typical applications of adaptive filtering are system identification, echo cancelation, signal prediction, noise elimination, etc.

By introducing the impulse response coefficient error vector:

$$
\boldsymbol{c}(n) \triangleq \boldsymbol{w}_{0}-\widehat{\boldsymbol{w}}(n)
$$

The mean square coefficient deviation at convergence state is [7]:

$$
\begin{aligned}
& \lim _{n \rightarrow \infty} \mathrm{E}\left\{\mathbf{c}(n)^{2}\right\}=\frac{\beta N \sigma_{v}^{2}}{\left(2-\beta N \sigma_{x}^{2}\right)}- \\
& -\frac{2 \beta^{2}\left(1-\beta N \sigma_{x}^{2}\right) N \sigma_{x}^{2} \sigma_{v}^{2} \sum_{m=1}^{M-1}(M-m)\left(1-\beta N \sigma_{x}^{2}\right)^{m-1}}{2 \beta M \sigma_{x}^{2}+\beta^{2} N M \sigma_{x}^{4}}
\end{aligned}
$$

Where

$\beta \triangleq \mu /\left(N \sigma_{x}^{2}+\delta\right)$

And $\sigma_{x}^{2}$ is the input signal variance and $\sigma_{v}^{2}$ is the noise variance.

\section{ENHANCED IMPULSE REASPONSE MEASUREMENT THROUGH APA}

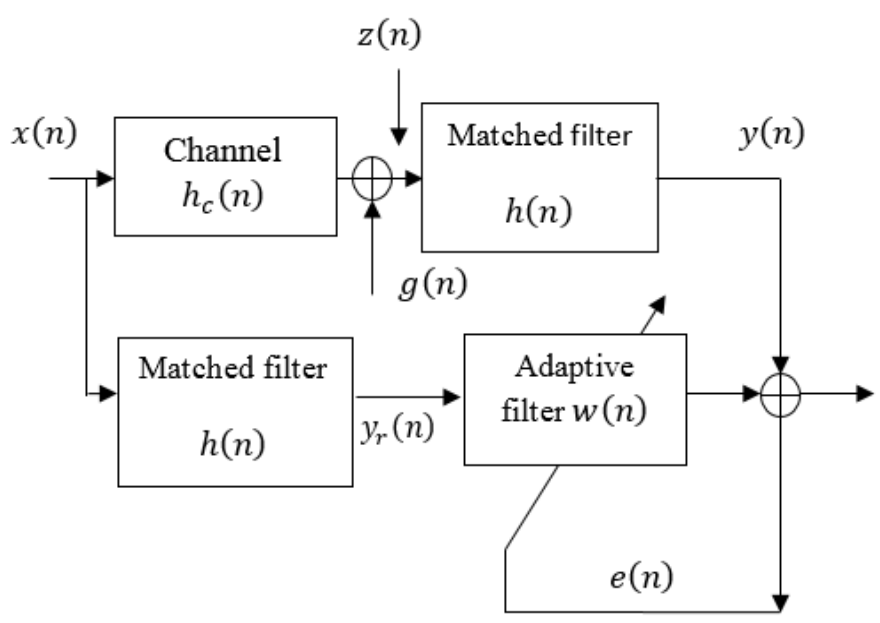

Fig-7: Block diagram of the enhanced matched filtering

Our proposed enhancement, for a classical matched filtering application, is presented schematically in Fig-7 and it presumes the existence of a second branch in the signal processing algorithm, through which it passes the same input signal as in the first branch, but this time, first it passes directly into the matched filter and the output goes into an adaptive filter which adapts based on the difference $\mathbf{e}(n)$ between the output of the first branch and the output of the adaptive branch. In the end, at convergence state the adaptive filter will have the same impulse response with the Channel.

The input signal is $x(n)$, a chirp with a sweep frequency from $\mathrm{f}_{1}$ to $\mathrm{f}_{2}$ and the analyzed signal will be

$$
z(n)=\sum_{i=1}^{N} A_{i} x\left(n-n_{i}\right)+g(n)
$$

Which is obtained based on the following filtering

$z(n)=\left(x * h_{c}\right)(n)+g(n)$

where

$h_{c}(n)=\sum_{i=1}^{N} A_{i} \delta\left(n-n_{i}\right)$

$h_{c}(n)$ represents the impulse response of the analyzed channel, and $g(n)$ is a white Gaussian noise that models the noise it has variance $\sigma^{2}, \mathrm{~N}$ is the length of the impulse response.

The adaptive filtering in based on an APA algorithm, at convergence state

$w(n) \cong h_{c}(n)$ 


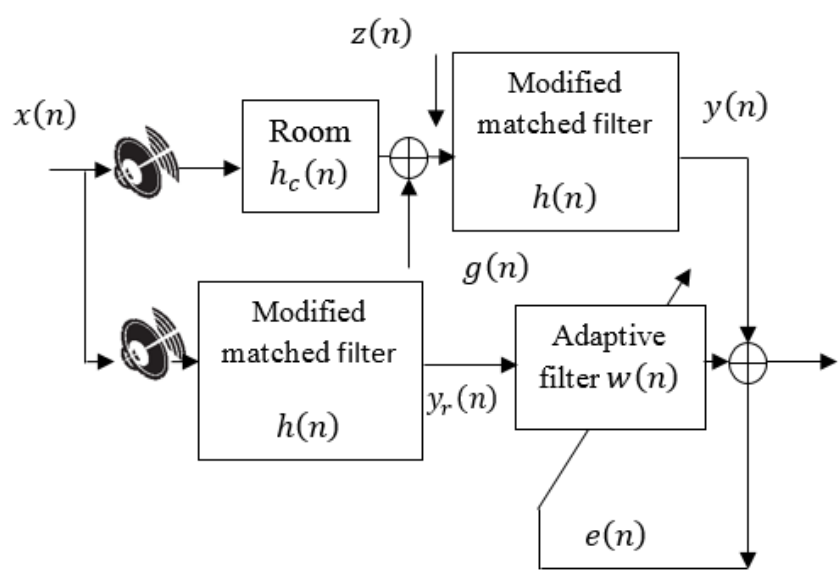

Fig-8: Block diagram of the enhanced sine sweep algorithm including loudspeaker nonlinearities

Same principle can be applied in a room impulse response measurement through sine sweep situations, and the block diagram from Fig-7 turns to the block diagram presented in Fig-8. Instead of using $x(n)$ in (14)-(15) it will be used $x_{d}(n)$, see (3), a signal based on $x(n)$, generated with (2), that incorporates the nonlinearities due to the loudspeaker.

\section{NUMERICAL RESULTS}

\section{Numerical results for matched filtering applications}

For the first scenarios we used a simple linear filter of length 100 , with only 4 coefficients different from zero and sampling frequency $2000 \mathrm{~Hz}, \mathrm{f}_{1}=10 \mathrm{~Hz}, \mathrm{f}_{2}$ $=100 \mathrm{~Hz}, x(n)$ is a chirp signal.
In Fig-9 we can compare visually the proposed algorithm in contrast with the classical matched filtering method, as it can be seen the proposed method behaves better. If we compare numerically the differences using MSE (mean square error) we obtain the following results:

- $\mathrm{MSE}_{\text {sine_sweep }}=-12.67 \mathrm{~dB}$;

- $\mathrm{MSE}_{\text {sine_sweep+adaptive filter }}=-58.47 \mathrm{~dB}$.

$\operatorname{MSE} \square \frac{1}{N} \sum_{n=1}^{N}\left[\mathrm{~h}_{\mathrm{c}}(\mathrm{n})-\mathrm{h}_{\mathrm{c} \__{-} \text {Ext }}(\mathrm{n})\right]^{2}$

In this simple scenario, the proposed method improves the MSE of the measurement with about $36 \mathrm{~dB}$. In this first case the signal that passes through the room is not affected by noise. Also, the resolution it is improved in matched filtering applications if we use an enhancement based on adaptive filtering. (See Fig-7)

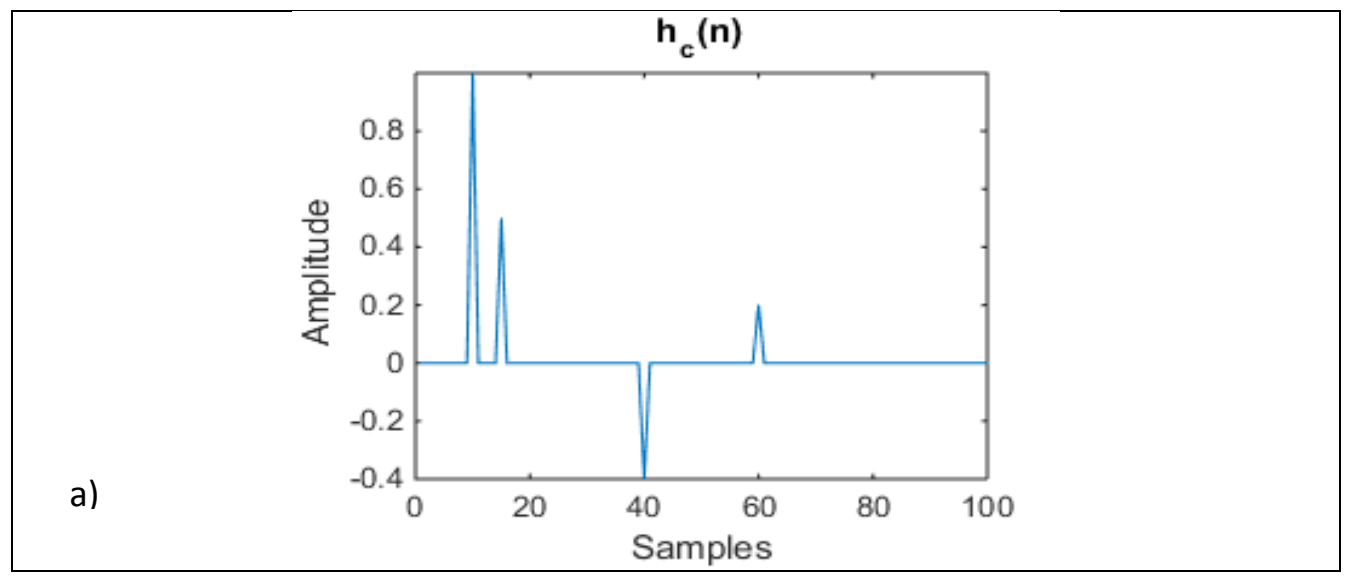




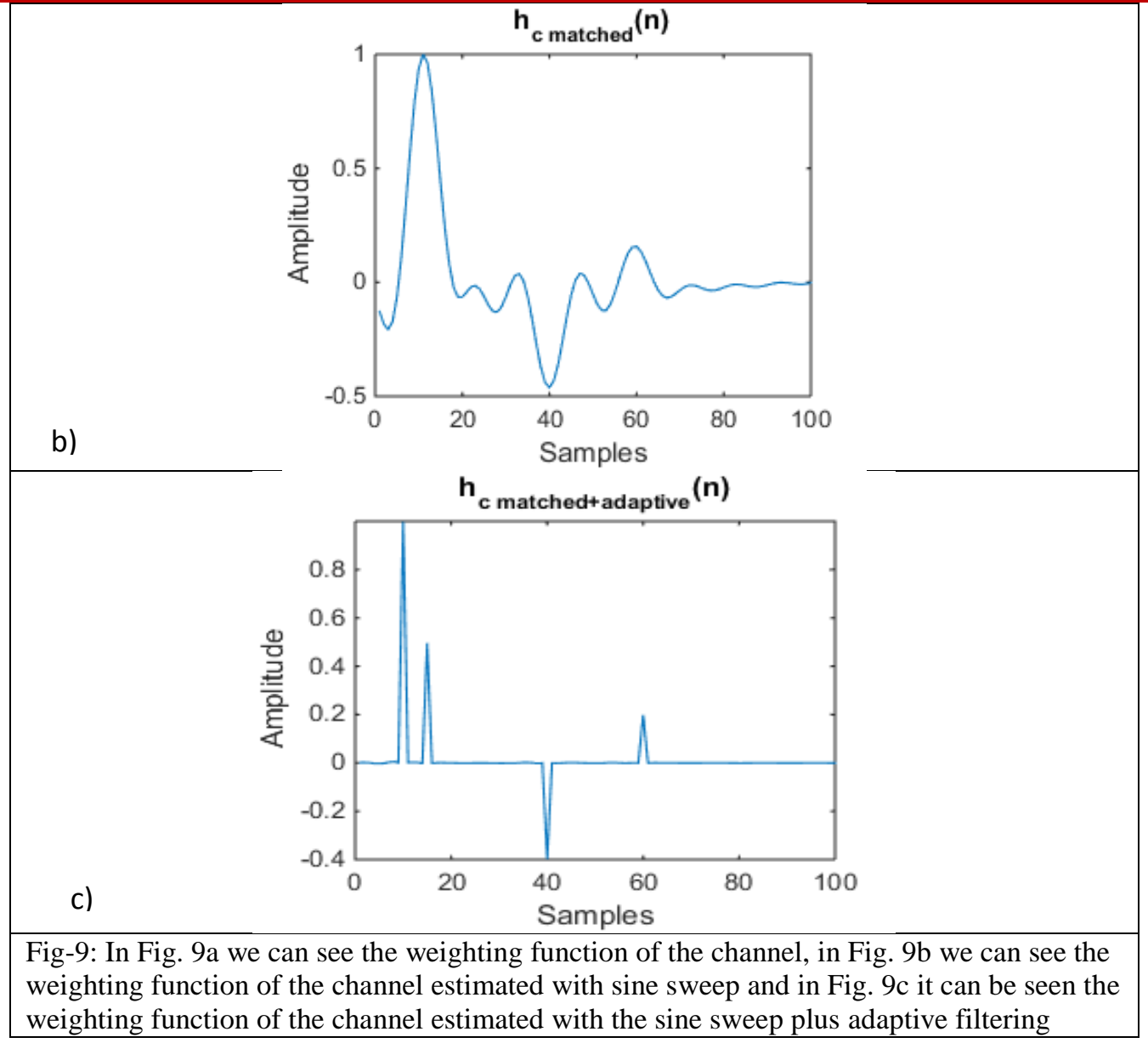

\section{Emulating loudspeaker nonlinearities}

For the second scenarios we simulate the conditions met in a sine sweep impulse response measurement application. We use the impulse response of a room with reverberation time of about $0.3 \mathrm{~s}$ and we also model the nonlinearities of the loudspeaker. The parameters for the APA are $M=4, \mu=1, \delta=0.1$. The block diagram presented in Fig- 8 was emulated using Matlab.

The loudspeaker nonlinearities were modeled using the Hammerstein model, see (3).

In Fig-10 we can compare the impulse response obtained using classical sine sweep, the impulse response using the enhanced sine sweep algorithm, based on Fig-8, with the real impulse response of the room. Also, for this experiment we simulated the nonlinearities of the loudspeaker that are involved in a real case scenario.

By computing MSE between the real impulse response and the impulse responses obtained with the classical sine sweep algorithm and with the proposed enhancement we obtained:

- $\mathrm{MSE}_{\text {sine_sweep }}=-43.67 \mathrm{~dB}$;

- $\mathrm{MSE}_{\text {sine_sweep+adaptive filter }}=-52.46 \mathrm{~dB}$.
The proposed method obtained an improvement in the MSE of about $9 \mathrm{~dB}$. But in this case we assumed that the noise $\mathrm{g}(\mathrm{n})$ is 0 . This method has the disadvantage that it incorporates in the adaptive filter also the noise $\mathrm{g}(\mathrm{n})$, this way the SNR (signal to noise ratio) should be high to be able to obtain satisfactory measured impulse responses of rooms. In Table-1 it can be seen the behavior of the two algorithms (classical sine sweep and proposed method), regarding MSE. The proposed method can give results, at least as good as classical sine sweep method, if the SNR of the recorded signal is higher than $35 \mathrm{~dB}$, in normal situations is a value easy to achieve but in noisy environments it may be difficult to be achieved.

We can see that the proposed enhancement compensates the nonlinearities of the loudspeaker.

Table-1: Performances of Sine Sweep Method and Proposed Method for Different SNRS

\begin{tabular}{|c|c|c|}
\hline SNR & MSE $_{\text {SINE_SWEEP }}$ & MSE $_{\text {SWEEP+ADAPTIVE }}$ \\
\hline $\mathbf{6 0}$ & -43.67 & -52.39 \\
\hline $\mathbf{5 5}$ & -43.67 & -52.17 \\
\hline $\mathbf{5 0}$ & -43.67 & -51.58 \\
\hline $\mathbf{4 5}$ & -43.67 & -49.31 \\
\hline $\mathbf{4 0}$ & -43.67 & -46.18 \\
\hline $\mathbf{3 5}$ & -43.67 & -43.35 \\
\hline $\mathbf{3 0}$ & -43.61 & -38.49 \\
\hline
\end{tabular}




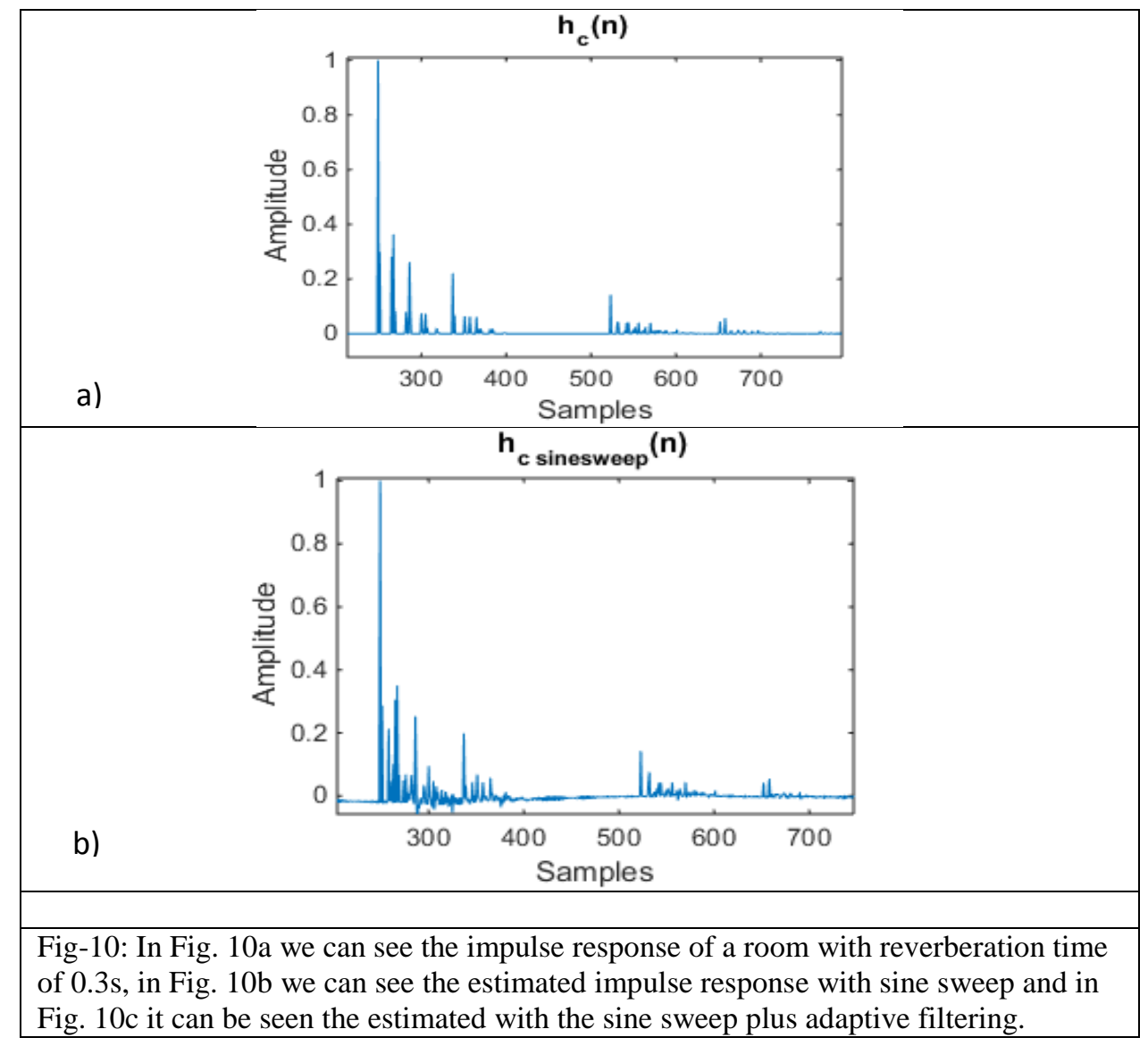

\section{CONCLUSIONS}

In this paper, we have developed and enhanced sine sweep algorithm, using adaptive filtering, for improving room impulse response measurements.

To quantify the performances of the proposed method we created two scenarios, in one scenario we tested the method for a classical matched filtering application with input signal a chirp and a simple impulse response, of length 100 , and with only 4 coefficients different from zero. By comparing MSE between the real impulse response and the measured one, based on classical matched filtering and on the proposed enhanced version, an improvement of about $36 \mathrm{~dB}$ was found and an improvement regarding the resolution of the measurement was obtained. To mention that for this first scenario we assumed that the noise that could affect the measurement was zero.

In the second scenario we simulate the conditions met in a sine sweep measurement situation, we use an impulse response of a room with reverberation time $0.3 \mathrm{~s}$ and we also have taken into consideration the nonlinearities of the loudspeaker and the noise that could affect the measurement. We found that the proposed enfacement may work better than the classical sine sweep method if a SNR greater than $35 \mathrm{~dB}$ can be assured. At a
SNR of about $50 \mathrm{~dB}$ the proposed method improves MSE with almost $8 \mathrm{~dB}$ in comparison with the classical sine sweep method. The proposed enhancement compensates the nonlinearities of the loudspeaker.

Some improvements regarding the behavior of the proposed enhanced sine sweep method in noisy conditions may be developed if we conduct further research regarding the use of a noise cancellation block.

\section{ACKNOWLEDGEMENTS}

The work has been funded by the Operational Programme Human Capital of the Ministry of Europe Funds through the Financial Agreement 51675/09.07.2019, SMIS code 125125.

\section{REFERENCES}

1. Farina, A. (2007, May). Advancements in impulse response measurements by sine sweeps. In Audio Engineering Society Convention 122. Audio Engineering Society.

2. Craven, P. G., \& Gerzon, M. A. (1992, September). Practical adaptive room and loudspeaker equaliser for HI-FI use. In Audio Engineering Society Conference: UK 7th Conference: Digital Signal Processing (DSP). Audio Engineering Society. 
Valentin Adrian Niță; Saudi J Eng Technol, Jan, 2021; 6(1): 9-16

3. Griesinger, D. (1996, November). Beyond MLSOccupied hall measurement with FFT techniques. In Audio Engineering Society Convention 101. Audio Engineering Society.

4. Müller, S., \& Massarani, P. (2001). Transferfunction measurement with sweeps. Journal of the Audio Engineering Society, 49(6), 443-471.

5. Stan, G. B., Embrechts, J. J., \& Archambeau, D. (2002). Comparison of different impulse response measurement techniques. Journal of the Audio Engineering Society, 50(4), 249-262.
6. Schmitz, T., \& Embrechts, J. J. (2016, September). A new toolbox for the identification of diagonal volterra kernels allowing the emulation of nonlinear audio devices. In International Congress on Acoustics: ICA, Argentine 5-9 Septembre 2016.

7. Dobre, R. A., Niţă, V. A., Ciochină, S., \& Paleologu, C. (2015, July). New insights on the convergence analysis of the affine projection algorithm for system identification. In 2015 International Symposium on Signals, Circuits and Systems (ISSCS) (pp. 1-4). IEEE. 